dan und H. Meckel mit der praktischen Anwendung bei der Redaktion von Kartenwerken, bei der Erstellung von Registern und beim Aufbau toponymischer Datenbanken. Die gelungene Veranstaltung war von Vertretern verschiedener Disziplinen besucht, die auch Gelegenheit fanden, den Austausch untereinander zu pflegen.

Am 11.10.1996 fand die Exkursion in das Burgenland statt, die sich der Thematik des vorangegangenen Symposiums anschloß, denn dort leben ungarische und kroatische Minderheiten, die seit jeher das Interesse der österreichischen Sprachforschung beanspruchen. Die Fahrt war durch die Veranstalter und nicht zuletzt mit Hilfe von leitenden Beamten der Burgenländischen Landesregie- rung bestens vorbereitet worden. Besucht wurden die Stadt Oberpullendorf und die Gemeinde Großwarasdorf, wo die Bürgermeister und weitere Experten über die Situation der jeweiligen Minderheiten referierten und zahlreiche Fragen kompetent beantworteten. Es zeigte sich dabei, daß nicht nur die Entstehung der Sprachinseln sehr unterschiedlich verlief, sondern daß auch die heutige Lage in wesentlichen Situationen voneinander abweicht. Die Exkursion schloß mit einem Besuch des Klosters Klostermarienberg, wo eine Ausstellung über die Siedlungstätigkeit und das Leben der Zisterzienser das Interesse der Teilnehmer in Anspruch nahm.

Walter Sperling, Trier

\title{
HMRSC IV - Viertes internationales Symposium über Fernerkundung in der Hochgebirgskartographie
}

Nicht nur in Kapitel 13 der Agenda 21 der Vereinten Nationen "Managing Fragile Ecosystems: Sustainable Mountain Development“, spiegelt sich die zunehmende Bedeutung der Bergregionen der Welt wider: Bereits 1990 versammelten sich über eine Initiative des Berichterstatters in Schladming in der Steiermark Fachleute aus vier Kontinenten, um nach Beijing/Lhasa 1992 und Mendoza (Argentinien) 1994 im Rahmen von High Mountain Remote Sensing Cartography (HMRSC) an der vierten Veranstaltung dieser Reihe in Schweden teilzunehmen:

Rund 45 Experten aus 15 Ländern in Südamerika, Nordamerika, Afrika, Asien und Europa trafen sich am 19. August 1996 zu einem dreitägigen Vortragsprogramm an der Universität Karlstad, einem der skandinavischen Zentren der Hochgebirgsfernerkundung, an welches eine achttägige Fachexkursion nach Lappland anschloß.

Die Beiträge der wissenschaftlichen Sitzungen reichten wie bei früheren Veranstaltungen von mehr theorieorientierten, methodischen Präsentationen bis zu sehr anwendungsbezogenen Erfahrungsberichten über Fallstudien. Vom Einsatz der Wavelet-Transformation über solare Einstrahlungsmodelle, Erosions-, Erdrutsch- und Permafrostkartierung bis zur Regionalplanung und Habitatbewertung für Wildtierarten reichte das Spektrum. Als genereller Trend ist der zunehmende Einsatz von GIS und GPS sowie die vermehrte Analyse von Fernerkundungszeitreihen zur Untersuchung dynamischer Phänomene zu nennen.

Während der Exkursion konnte im Rahmen der Feldstudien im Torneträsk-Gebiet westlich von Kiruna der Bezug zu den Vortragsveranstaltungen hergestellt werden. Ausgerüstet mit topographischen und thematischen Karten sowie mit eigens für HMRSC IV produzierten Landsat-TM- und ERS-SAR-Szenen ging es in den Abisko-Nationalpark mit der dort befindlichen Forschungsstation und in das geologisch-geomorphologisch hochinteressante Kärkevagge (Kärke-Tal). Besuche in Kiruna waren dem Environmental Satellite Data Center, der ESA-Satellitenstation, dem Esrange Space Operations Centre sowie den Firmen Swedish Space Corporation - Satellitbild und Metria gewidmet. Eindrucksvoll gestaltete sich der Wandertag von der schwedisch-norwegischen Grenze, dem Ort Riksgränsen, hinunter zum Rombaksfjord, von wo es per Speed-Katamaran und Linienschiff der Hurtiglinie über Narvik und die Lofoten nach Tromsö ging. Dort wurde über die in fruchtbarer Kooperation von Universität und Industrie durchgeführten Aktivitäten auf den Gebieten der Weltraumforschung und Fernerkundung berichtet sowie das Roald Amundsen Center for Arctic Research und das Planetarium der Universität, weiters die Tromsö Satellite Station besucht.

Die Symposiumsbeiträge werden als Tagungsband und als Electronic Proceedings im Internet (dort mit farbigen Abbildungen) erscheinen. Dafür sowie für die vorbildliche Organisation und reibungslose Durchführung dieser komplexen Veranstaltung sei Dr. Gerhard Bax von der Universität Karlstad, der dieses vierte Symposium von HMRSC zur vollen Zufriedenheit aller Teilnehmer zum Abschluß bringen konnte, herzlich gedankt.

Manfred Buchroithner, Dresden 